\title{
Strong pairwise ant-plant mutualism has limited spillover effects on an ant community
}

\author{
Marion L Donald ${ }^{1}$ and Tom Miller ${ }^{2}$ \\ ${ }^{1}$ Manaaki Whenua \\ ${ }^{2}$ Rice University
}

September 24, 2021

\begin{abstract}
Pairwise mutualisms are embedded within rich communities of co-occurring species. Mutualism by definition benefits partner species and theory predicts these effects can spill over into broader communities, with consequences for abundance, diversity, and composition. Specifically, positive feedbacks between partner species may shift competitive hierarchies and drive reductions in non-partner species. We used long-term monitoring data spanning 16 years to determine the ant partner species of tree cholla cacti (Cylindriopuntia imbricata), which reward ants with extrafloral nectar in exchange for anti-herbivore defense. We coupled these long-term data with short-term characterization of the ant community via pitfall trapping, which sampled partner and non-partner species across ten plots that varied naturally in cactus density. The long-term data revealed one dominant ant partner (Liometopum apiculatum) and two other less common partners (Crematogaster opuntiae and Forelius pruinosus). In the short-term census, the dominant ant partner occupied more cacti in plots of higher cactus density, and was also found at higher occurrence within the pitfall traps in the high cactus density plots, suggesting strong positive feedbacks that promote ant occurrence where plant partners are available. Despite the consistency of L. apiculatum partnership with cacti through time and the dominance of this partnership in areas of high cactus density, spillover effects from this mutualism appear limited. Of the common ant species, a single non-partner ant species showed a modest reduction in occurrence at high cactus density, possibly in response to increased presence of L. apiculatum. Additionally, the composition and diversity of the ant community in our plots were insensitive to cactus density variation, indicating that positive effects on the dominant ant partner did not have cascading impacts on the ant community. This study provides novel evidence that pairwise mutualisms, even those with strong positive feedbacks, may be limited in the scope of their community-level effects.
\end{abstract}

\section{Hosted file}

Manuscript.pdf available at https://authorea.com/users/435180/articles/538377-strongpairwise-ant-plant-mutualism-has-limited-spillover-effects-on-an-ant-community 